\title{
High Resolution Simulation of Nonstationary Gaussian Random Fields
}

\author{
William Kleiber ${ }^{1}$
}

January 21, 2016

\begin{abstract}
Simulation of random fields is a fundamental requirement for many spatial analyses. For small spatial networks, simulations can be produced using direct manipulations of the covariance matrix. Larger high resolution simulations are most easily available for stationary processes, where algorithms such as circulant embedding can be used to simulate a process at millions of locations. We discuss an approach to simulating high resolution nonstationary Gaussian processes that relies on generating a stationary random field followed by a nonlinear deformation to produce a nonstationary field. A spatially varying variance coefficient accounts for local scale effects. The nonstationary covariance function is estimated nonparametrically, and the deformation function is then estimated in a variational framework. We illustrate the proposed approach on synthetic datasets, a challenging temperature dataset over the state of Colorado and a regional climate model over North America.
\end{abstract}

Keywords: Circulant embedding; Deformation; nOnstationary; SimulaTION; STATIONARY; WARPING

\section{Introduction}

Spatial analyses typically involve three common goals: the first is surface estimation based on incomplete or noisy observations, the second is interpretation of a spatial model to gain scientific insight of a particular process, and the third is simulation. Simulation plays an

\footnotetext{
${ }^{1}$ Department of Applied Mathematics, University of Colorado, Boulder, CO. Author e-mail: william.kleiber@colorado.edu
} 
important role for both of the first two goals; in surface estimation simulation can be used to quantify predictive uncertainty via conditional simulation and can also yield field realizations consistent with a partially observed process. In the second goal, simulation can give insight into the statistical properties of a process, such as its spatial length scale, smoothness, level crossings or extrema.

Suppose interest focuses on simulating a random process $Z(\mathbf{s}), \mathbf{s} \in \mathbb{R}^{d}$. Without loss of generality we assume $Z(\mathbf{s})$ is a mean zero process; otherwise we would simulate a mean zero field onto which we add a nontrivial mean function. We additionally assume $Z(\mathbf{s})$ is a Gaussian process, whose stochastic behavior is then fully known once the covariance function $C\left(\mathbf{s}_{1}, \mathbf{s}_{2}\right)=\operatorname{Cov}\left(Z\left(\mathbf{s}_{1}\right), Z\left(\mathbf{s}_{2}\right)\right)$ is specified.

Simulation of random fields can be either unconditional or conditional. Unconditional simulation is simply direct simulation of the process $Z(\mathbf{s})$. Conditional simulation, on the other hand, is the simulation of $Z(\mathbf{s})$ conditioned on some observations in order to generate plausible realizations that are consistent with the partially observed process. In this manuscript we mainly focus on unconditional simulation, noting that conditional simulation can be directly formulated via unconditional simulations (Journel, 1974).

It seems there are at least three possible ways to simulate nonstationary field - first, use a stochastic model that allows for nonstationarity and directly simulate from this (e.g., a nonstationary Matérn); this requires a Cholesky decomposition of the covariance matrix which is infeasible for large simulation grids. The second is to explicitly build such a process from a set of basis functions, e.g., using a Karhunen-Loéve expansion or polynomial chaos expansion. The final method is one explored in this manuscript, where a mapping is 
developed between the nonstationary process and a stationary process, whence simulations involve only the straightforward stationary generation. We note that there is some literature on simulation methods for nonstationary intrinsic random functions, although we do not focus on these here (Stein, 2001, 2002, and references therein).

Our proposal relies on the spatial deformation approach of Sampson and Guttorp (1992) to achieve a mapping between the nonstationary and stationary plane. The basic idea is that in the stationary plane high resolution simulations are easy to produce from some existing method such as circulant embedding, and then the inverse transformation results in a high dimensional nonstationary simulation. Note we are primarily concerned with simulation, whereas Sampson and Guttorp (1992) and ensuing literature has focused almost exclusively on modeling and kriging. The type of nonstationarity that can be captured by deformation is either known as stationary isotropic reducible or stationary reducible (Perrin and Senoussi, 1999, 2000). Deformation has successfully been used to capture a large number of physical processes (Guttorp et al., 1992; Monestiez et al., 1993; Guttorp and Sampson, 1994; Brown et al., 1994; Guttorp et al., 1994; Meiring, 1995), but not all types of nonstationarity can be reduced to stationarity this way, e.g., a Matérn covariance with spatially varying smoothness cannot be (Stein, 2005; Paciorek and Schervish, 2006). Note some similarities with the timedeformation method in economics (Barndorff-Nielsen and Shepard, 2006).

We consider two motivating environmental examples: the first is application in the field of stochastic weather generators, while the second involves statistical emulation or analysis of a regional climate model. Stochastic weather generators are probabilistic models whose simulations behave statistically similarly to observations (Wilks and Wilby, 1999). These 
simulators are used primarily in the hydrologic and climate sciences to perform downscaling or impact assessments (Semenov and Barrow, 1997). Typically, weather realizations are required on a grid, sometimes requiring simulation over very large geographical regions at high resolutions (Serinaldi and Kilsby, 2014). Following the technical development of our approach, we illustrate its implementation on a challenging temperature dataset over the state of Colorado. For the second example, we consider the problem of stochastically simulating fields of regional climate model (RCM) output that are consistent with RCM runs. These products are crucial for climate forecasting or model emulation, that is, using a statistical model as a fast surrogate for a computationally expensive physical climate model. RCMs are usually run coupled with a general circulation model (GCM) in order to better represent local nonstationarities that are driven by local geographical effects that are not well represented in a coarse GCM grid. The ability to quickly generate stochastic realizations from a high resolution nonstationary process is of fundamental importance for both of these applications.

\section{Simulation of Random Fields}

Our approach to nonstationary random field simulation relies on fast simulation algorithms for stationary or isotropic random fields. We begin this section with a brief overview of some classic algorithms for stationary simulation, Schlather (2012) and Kroese and Botev (2013) give nice recent overviews of some of these approaches among others. 


\subsection{Stationary Simulation}

Momentarily suppose the random field $Z(\mathbf{s})$ is stationary, that is, $C\left(\mathbf{s}_{1}, \mathbf{s}_{2}\right)=C\left(\mathbf{s}_{1}-\mathbf{s}_{2}\right)$ is a function of the lag vector separating two spatial locations. The spectral method is a traditional approach to approximately simulating stationary random fields. The spectral method (Shinozuka and Jan, 1972) relies on using the spectral representation of a random field requiring continuity of the covariance function,

$$
Z(\mathbf{s})=\operatorname{Re}\left[\int \exp \left(2 \pi 1 \boldsymbol{\omega}^{\prime} \mathbf{s}\right) \mathrm{d} Y(\boldsymbol{\omega})\right]
$$

where $\boldsymbol{\omega} \in \mathbb{R}^{d}$ and $\mathrm{d} Y(\boldsymbol{\omega})$ is a complex-valued Gaussian measure with zero mean and whose pointwise variance is $\mathcal{F}(C)(\boldsymbol{\omega}) \mathrm{d} \boldsymbol{\omega}, \mathcal{F}$ denoting the Fourier transform. Simulations of $Z(\mathbf{s})$ can then be approximately generated by using a discrete approximation to the integral representation (1). The turning bands method, originally suggested by Matheron (1973), relies on simulating a multidimensional isotropic Gaussian random field by summing simulations from one-dimensional processes that have been embedded in more than one dimension (Mantoglou and Wilson, 1982; Dietrich, 1995; Gneiting, 1996, 1999). In particular, realizations for $d=2,3$ are obtained via

$$
Z(\mathbf{s})=\frac{1}{\sqrt{L}} \sum_{i=1}^{L} Z_{i}\left(\mathbf{s} \cdot \mathbf{e}_{i}\right)
$$

where $\left\{Z_{i}(\cdot)\right\}_{i=1}^{L}$ are mutually independent one-dimensional processes, $\left\{\mathbf{e}_{i}\right\}_{i=1}^{L}$ are unit vectors and - indicates the dot product. The key difficulty turns out to be identifying the one-dimensional covariance function that corresponds to the desired multidimensional covariance structure, with the most common two-dimensional case requiring solving an Abel 
integral equation (Gneiting, 1998). For stationary fields whose covariance can be represented as a convolution, $C(\mathbf{h})=\int g(\mathbf{s}) g(\mathbf{s}+\mathbf{h}) \mathrm{d} \mathbf{s}$, one can use the so-called random coin (or dilution) method to generate approximate realizations (Chilès and Delfiner, 1999; Schlather, 2012). Ehm et al. (2004) examined conditions on compactly supported covariance functions that admit such a convolution representation. The approximate simulation is $\sum_{\mathbf{h} \in \Pi} g(\mathbf{s}-\mathbf{h})$ where $\Pi$ is a stationary Poisson point process with unit intensity; in practice one sums multiple simulations and then relies on the central limit theorem to generate approximate stationary Gaussian process realizations. Another approximate simulation method is based on a propagative Gibbs sampler (Lantuéjoul and Desassis, 2012) which only requires univariate normal samples at every step. However, each univariate normal is is based on computing simple kriging estimates at each iteration, and can be computationally time consuming.

A direct approach to simulation is to specify the stochastic process through an explicit model. For example, Lindgren et al. (2011) use stochastic partial differential equations to specify a spatial process, and whose simulations then are essentially based on a basis function decomposition. Similarly, Nychka et al. (2014) introduced the LatticeKrig approach, another basis decomposition idea, in which the process is broken up into differing levels of resolution; simulation in both of these cases is straightforward given the specification of the process.

Probably the most popular modern method for stationary random field simulation is circulant embedding (Dietrich and Newsam, 1993, 1997; Wood and Chan, 1994), in part due to the fact that the spectral, turning bands, random coin and Gibbs methods only generate approximate realizations. For two-dimensional simulation, if simulation points are on a regular grid then the corresponding covariance matrix is block Toeplitz. By appropriately 
embedding this block Toeplitz matrix into a circulant matrix $\Sigma$, we can decompose $\Sigma=$ $F D F^{*}$ where $D$ is a diagonal matrix of eigenvalues of $\Sigma$ and $F$ has complex exponential entries corresponding to the fast Fourier transform (FFT). The key to simulation is that FFT techniques can be applied to decompose the circulant matrix easing computational burden. If the circulant matrix has negative eigenvalues then this approach fails. Gneiting et al. (2006) explore two alternatives, cut-off and intrinsic embedding, both suggested by Stein (2002), as remedies for this issue that still yield exact simulations.

\subsection{Nonstationary Simulation}

Apart from intrinsic random functions and related constructions (Stein, 2001), less attention has been directed toward efficient nonstationary process simulation. Some approaches involve analogues of the spectral method (Deodatis, 1996; Liang et al., 2007) and FFTs ap-

plied to adapted spectra ( $\mathrm{Li}$ and Kareem, 1991), but these seem to be mostly relegated to the engineering literature. Other promising approaches (that, too, have not seen much attention in the statistics literature), involve Karhunen-Loéve expansions (Huang et al., 2001; Spanos et al., 2007; Sarma et al., 2008), polynomial chaos approaches (Ghanem and Spanos, 1990; Sakamoto and Ghanem, 2002a,b; Soize and Ghanem, 2004), or general basis function expansions (Puig et al., 2002). Some approaches can additionally allow for non-Gaussian simulation (Deodatis and Micaletti, 2001). We now suppose $Z(\mathbf{s})$ is a possibly nonstationary process.

One relatively straightforward way to simulate a general nonstationary Gaussian random field at locations $\mathbf{s}_{i}, i=1, \ldots, n$ is via matrix manipulations. If $\Sigma$ is the covariance matrix 
of the vector $\left(Z\left(\mathbf{s}_{1}\right), \ldots, Z\left(\mathbf{s}_{n}\right)\right)^{\prime}$ and has Cholesky decomposition $\Sigma=T T^{\prime}$ where $T$ is lower triangular, then the random vector $T\left(\varepsilon_{1}, \ldots, \varepsilon_{n}\right)^{\prime}$ also has covariance matrix $\Sigma$, where $\varepsilon_{i}$ are uncorrelated standard normal random variables . For large $n$, simulation and storage via the Cholesky decomposition for an unstructured and dense matrix $\Sigma$ is expensive, requiring $6 n^{5}$ flops, whereas circulant embedding for a stationary simulation requires $40 n^{2} \log _{2} 2 n$ (Dietrich, 1993; Gneiting et al., 2006).

The basic idea in this article is to apply an appropriate transformation to the nonstationary $Z(\mathbf{s})$ process so that the transformed process is stationary, whence we can exploit the fast simulation algorithms for stationary processes. The nonstationary simulation is then formed by back-transforming the stationary simulation. We generalize the approach of Sampson and Guttorp (1992) to allow for locally nonstationary variances, incorporate stationary simulations and update the estimation approach to focus on nonstationary versions of the covariance function, rather than the variogram.

The notion of spatial deformation for nonstationary modeling has been exploited by many authors for over two decades, and our proposal can be viewed as an extension of these ideas for the purposes of simulation. Sampson and Guttorp (1992) originally introduced the idea of using spatial deformations for nonstationary modeling. Since then, many authors have considered variations on this theme: space-time warping was considered by Meiring et al. (1997) and Meiring et al. (1998); Perrin and Monestiez (1998) introduced radial basis deformations to ensure bijectivity; Iovleff and Perrin (2004) discussed estimation via simulated annealing while Damian et al. (2001) provided a Bayesian approach. Some authors have considered theoretical implications of deformation, Perrin and Meiring (1999) show the de- 

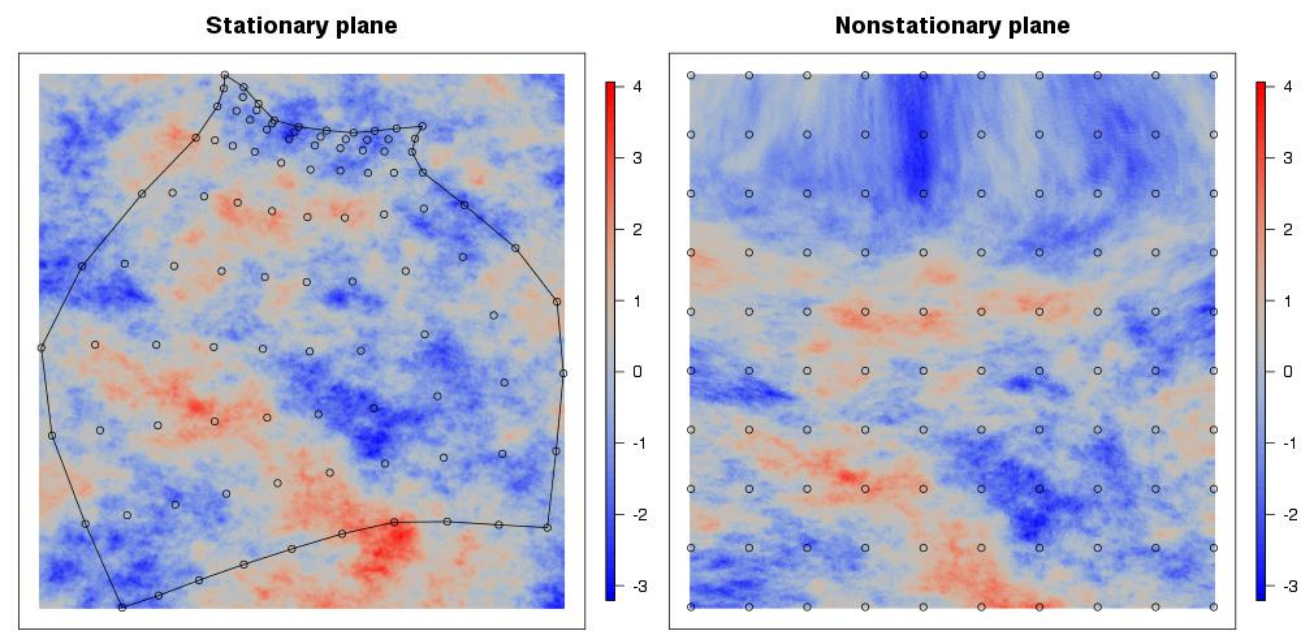

Figure 1: Example of approach: a stationary simulation is generated and then deformed to obtain a nonstationary random field realization.

formation model is unique under certain assumptions regarding the isotropic variogram in the stationary plane; Anderes and Stein (2008) introduced a new approach to deformation depending on orientation preserving diffeomorphisms and Anderes and Chatterjee (2009) discussed consistency of estimating deformations in an infill asymptotic framework. Sampson (2010) gives a recent overview.

Before considering the details of implementation, Figure 1 illustrates the proposed approach. The stationary simulation in the left panel is regularized by the overlaid deformation function, resulting in the nonstationary simulation on the right. For this example, the simulated values near the top of the deformed domain are stretched vertically, while values near the bottom retain approximate stationarity. Note that if the stationary process is Gaussian, then the nonstationary process (generated by spatially transforming the stationary process) will still be Gaussian, as it is just a re-indexed version thereof.

A useful representation for a nonstationary process is to explicitly separate variance from 
a spatially correlated process with unit variance, $Z(\mathbf{s})=\sigma(\mathbf{s}) Z_{0}(\mathbf{s})$. Here, $Z_{0}(\mathbf{s})$ has correlation function $R(\cdot, \cdot)$ and $\operatorname{Var} Z_{0}(\mathbf{s})=1$. Thus, $C\left(\mathbf{s}_{1}, \mathbf{s}_{2}\right)=\sigma\left(\mathbf{s}_{1}\right) \sigma\left(\mathbf{s}_{2}\right) R\left(\mathbf{s}_{1}, \mathbf{s}_{2}\right)$. We propose viewing the nonstationary process $Z_{0}(\mathbf{s})$ as a geographically transformed version of a stationary process. In particular, we suppose the existence of a mean zero stationary process $D(\mathbf{s})$ with correlation function $R_{D}(\mathbf{h})$ and an invertible deformation function $\varphi: \mathbb{R}^{d} \rightarrow \mathbb{R}^{d}$ such that $Z_{0}(\mathbf{s})=D(\varphi(\mathbf{s}))$, or equivalently $Z_{0}\left(\varphi^{-1}(\mathbf{s})\right)=D(\mathbf{s})$. Thus,

$$
\operatorname{Cov}\left(Z_{0}\left(\mathbf{s}_{1}\right), Z_{0}\left(\mathbf{s}_{2}\right)\right)=\sigma\left(\mathbf{s}_{1}\right) \sigma\left(\mathbf{s}_{2}\right) R_{D}\left(\varphi\left(\mathbf{s}_{1}\right)-\varphi\left(\mathbf{s}_{2}\right)\right) .
$$

If the goal is to simulate a vector $\left(Z\left(\mathbf{s}_{1}\right), \ldots, Z\left(\mathbf{s}_{n}\right)\right)^{\prime}$, this is achieved by simulating $\left(\sigma\left(\mathbf{s}_{1}\right) D\left(\varphi\left(\mathbf{s}_{1}\right)\right), \ldots, \sigma\left(\mathbf{s}_{n}\right) D\left(\varphi\left(\mathbf{s}_{n}\right)\right)\right)^{\prime}$. Simulation of $D$ is performed via a fast stationary technique jointly at locations $\left\{\varphi\left(\mathbf{s}_{i}\right)\right\}_{i=1}^{n}$; multiplication by $\sigma\left(\mathbf{s}_{i}\right)$ is simply a rescaling.

If the goal is to produce a gridded nonstationary simulation, there are two basic approaches. The first is to begin with a gridded stationary simulation and then deform it to the nonstationary plane - in this case the deformed version will almost never lay exactly on a grid, but an approximation to the nonstationary simulation can be performed by taking the nearest neighbor from the deformed stationary simulation to the nonstationary grid. The alternative approach is to begin by specifying the grid in the nonstationary domain, whence the deformation (e.g., that shown in Figure 1) results in an irregular grid in stationary space. There are then two roads: use a stationary simulation method that does not depend on regular spacing, such as the random coin or turning bands methods, or alternatively, approximate the irregular spacing with a very high resolution grid and perform a gridded stationary simulation such as with circulant embedding, then taking the nearest grid neighbor to the deformed irregular grid. In the examples below, we adopt this latter approach, 
and find in our experience that moderately dense stationary simulation grids work as well as extremely high resolution simulations.

\subsection{Estimation}

Based on the above discussion, there are two important functions to estimate: The first is the nonstationary process covariance $C\left(\mathbf{s}_{1}, \mathbf{s}_{2}\right)$, and the second is the geographical warping function $\varphi$.

Sampson and Guttorp (1992) work with spatial dispersions, which are nonstationary analogues of variogram values. Their basic idea was to estimate a monotone function of spatial distance to approximate the dispersions, followed by a invertible coordinate mapping to deform geographical space to a stationary plane. We take a slightly different approach by separately estimating the nonstationary covariance function, followed by estimation of the spatial deformation function to match a pre-specified stationary covariance. Either method requires separate estimation of the covariance (or dispersion), followed by optimization of the deformation. In a Bayesian context, one could consider joint estimation of the two in a conditional sampling framework, first estimating the covariance, then the deformation, followed by an acceptance-rejection step. However, this would require a flexible family of prior distributions for both classes of functions, which is beyond the scope of the current paper.

We begin by estimating the nonstationary spatial structure using a nonparametric estimator. In particular, if observations are available at spatial locations $\mathbf{s}_{1}, \ldots, \mathbf{s}_{n}$, then we 
form

$$
\hat{C}(\mathbf{x}, \mathbf{y})=\frac{\sum_{i=1}^{n} \sum_{j=1}^{n} K_{b}\left(\left\|\mathbf{x}-\mathbf{s}_{i}\right\|\right) K_{b}\left(\left\|\mathbf{y}-\mathbf{s}_{j}\right\|\right) Y\left(\mathbf{s}_{i}\right) Y\left(\mathbf{s}_{j}\right)}{\sum_{i=1}^{n} \sum_{j=1}^{n} K_{b}\left(\left\|\mathbf{x}-\mathbf{s}_{i}\right\|\right) K_{b}\left(\left\|\mathbf{y}-\mathbf{s}_{j}\right\|\right)}
$$

where $K_{b}$ is a kernel function with bandwidth $b$, and $\|\cdot\|$ denotes Euclidean norm. This nonparametric estimator has previously been employed and extended by numerous authors including Oehlert (1993), Guillot et al. (2001), Jun et al. (2011) and Kleiber et al. (2013), and whose theoretical properties were explored by Kleiber and Nychka (2012). This estimator has desirable properties as it is available at all spatial locations, and retains nonnegative definiteness. From this estimate, we extract the local variance estimates $\hat{\sigma}^{2}(\mathbf{s})=\hat{C}(\mathbf{s}, \mathbf{s})$ and nonparametric correlation function $\hat{R}\left(\mathbf{s}_{1}, \mathbf{s}_{2}\right)=\hat{C}\left(\mathbf{s}_{1}, \mathbf{s}_{2}\right) /\left(\hat{\sigma}\left(\mathbf{s}_{1}\right) \hat{\sigma}\left(\mathbf{s}_{2}\right)\right)$.

To estimate the deformation function $\varphi$ we pre-specify a stationary covariance from which the simulations will be generated, $R_{D}(\cdot)$. A straightforward way to estimate $\varphi$ is by minimizing a distance between the theoretical covariance and nonparametric estimator, for instance $\sum_{i=1}^{n} \sum_{j=1}^{n}\left(\hat{R}\left(\mathbf{s}_{i}, \mathbf{s}_{j}\right)-R_{D}\left(\varphi\left(\mathbf{s}_{i}\right)-\varphi\left(\mathbf{s}_{j}\right)\right)^{2}\right.$. However, minimizing such a criteria without restricting the class of functions $\varphi$ can lead to nonphysical behavior such as space folding upon itself. From a simulation perspective, this is an undesirable possibility, as this implies two spatial locations in undeformed space will necessarily have identical simulated values, and there are few situations in which this behavior is physically warranted. To this end, we follow Sampson and Guttorp (1992) by entertaining only particular classes of functions in a reproducing kernel Hilbert space $\mathcal{H}$. In particular, we estimate $\varphi$ by minimizing the penalized criterion

$$
\sum_{i=1}^{n} \sum_{j=1}^{n}\left(\hat{R}\left(\mathbf{s}_{i}, \mathbf{s}_{j}\right)-R_{D}\left(\varphi\left(\mathbf{s}_{i}\right)-\varphi\left(\mathbf{s}_{j}\right)\right)^{2}+\lambda\langle\varphi, \varphi\rangle\right.
$$


over $\varphi \in \mathcal{H}$ where $\mathcal{H}$ has corresponding inner product $\langle\cdot, \cdot\rangle$ and $\lambda \geq 0$ controls the amount of allowed deformation. Indeed, including the penalty term has the effect of regularizing the spatial deformation.

The spatial datasets we are interested in are indexed in the plane, $d=2$ dimensions. In this case, $\mathbf{s}=\left(s_{x}, s_{y}\right)^{\prime}$ and $\varphi=\left(\varphi_{x}, \varphi_{y}\right)^{\prime}$. In this case we use the penalty $\langle\varphi, \varphi\rangle=$ $\left\langle\varphi_{x}, \varphi_{x}\right\rangle+\left\langle\varphi_{y}, \varphi_{y}\right\rangle$ where each of these marginal inner products is

$$
\langle f, f\rangle=\int_{-\infty}^{\infty} \int_{-\infty}^{\infty}\left(\left(\frac{\partial^{2} f}{\partial s_{x}^{2}}\right)^{2}+2\left(\frac{\partial^{2} f}{\partial s_{x} \partial s_{y}}\right)^{2}+\left(\frac{\partial^{2} f}{\partial s_{y}^{2}}\right)^{2}\right) \mathrm{d} s_{x} \mathrm{~d} s_{y}
$$

so that $\mathcal{H}$ is the space of twice-differentiable functions with $\langle f, f\rangle<\infty$ (Bookstein, 1989; Green and Silverman, 1994). This type of penalty, resulting in a thin plate spline solution, has convenient algebraic expressions for computation, see Bookstein (1989) or Gilleland et al. (2011) for details. In this case, the two components of the vector-valued deformation function are expansions in terms of the radial basis function $R\left(\mathbf{s}, \mathbf{s}_{0 i}\right)=\left\|\mathbf{s}-\mathbf{s}_{0 i}\right\|^{2} \log \left(\left\|\mathbf{s}-\mathbf{s}_{0 i}\right\|^{2}\right)$,

$$
\varphi_{x}(\mathbf{s})=a_{0 x}+a_{1 x} s_{x}+a_{2 x} s_{y}+\sum_{i=1}^{L} b_{i x} R\left(\mathbf{s}, \mathbf{s}_{0 i}\right)
$$

with a similar form for $\varphi_{y}$; this basis function is intimately related to the form of the inner product $\langle\cdot, \cdot\rangle$, see Wahba (1990) for discussion. Sampson and Guttorp (1992) specify $L=n$ basis nodes centered at the observation locations, $\mathbf{s}_{0 i}=\mathbf{s}_{i}$. However, for moderate-to-large spatial datasets, this introduces a large number of statistical parameters defining the warp, and so setting $L<n$ is useful, where the locations $\left\{\mathbf{s}_{0 i}\right\}_{i=1}^{L}$ are user-specified landmark points, usually outlining important spatial features. 


\section{Data Illustrations}

We illustrate our proposed simulation approach in a number of idealized situations, with a challenging nonstationary meteorological dataset over complex terrain and in a climate model emulation context. Stationary simulations based on circulant embedding are performed in $\mathrm{R}$ using the RandomFields package (Schlather et al., 2014).

\subsection{Exploratory Examples}

The first example scenario involves the situation in which a process experiences regimedependent behavior. A prototypical example is modeling of temperature in and around valleys or orographic depressions where varying terrain implies non-ellipsoidal level curves of correlation. Alternatively this type of behavior is common at the boundary between land and water; typically authors separately model processes over land and ocean, while the proposed framework allows for simultaneous simulation over both regimes. For this motivating example, we simulate a stationary random field having Matérn correlation with unity smoothness and spatial range of five; the corresponding nonstationary simulation is shown in Figure 2 on a $500 \times 500$ grid. Note that such a simulation would be impossible to implement without restrictive assumptions on the covariance structure of the nonstationary process using the traditional matrix decomposition approach.

The second example we entertain is simulation of a vortex; this type of nonstationarity is common in hurricane modeling, for instance (Reich and Fuentes, 2007). Rotational nonstationarity is difficult to accommodate using extant models, and indeed it is unclear how high resolution simulation would proceed in a traditional fashion. Figure 3 shows an example 
Deformation

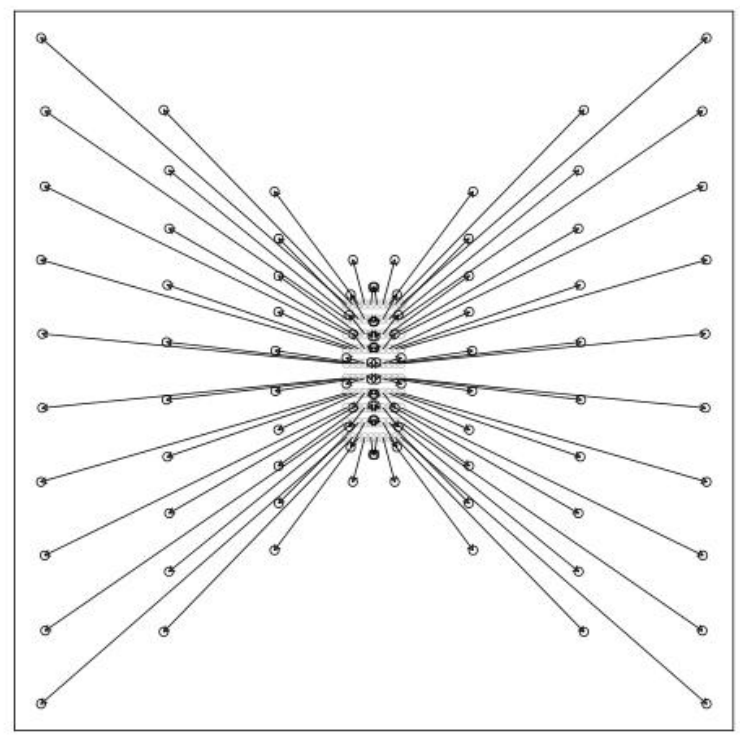

Simulation

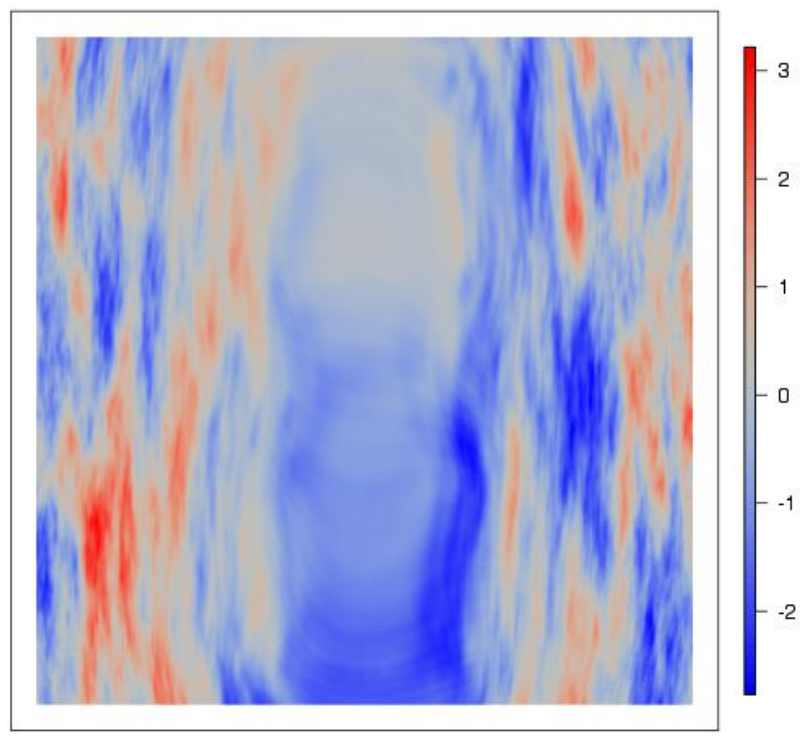

Figure 2: Valley simulation on a $500 \times 500$ grid. Simultaneous simulations between regime dependent climates are possible using the deformation approach.

simulation from a vortex model at 250,000 spatial locations, along with the associated deformation function. A particularly difficult quality to explicitly model (but which is present in our simulation) is the increased rotational velocity near the center of the vortex, while away from the center less rotation is apparent.

\subsection{High Resolution Temperature Simulation over Complex Ter- rain}

Stochastic weather generators (SWGs) are statistical models whose simulations statistically match observed weather patterns. Weather generators are particularly useful for downscaling and climate impact studies. Both of these goals often involve high resolution simulation of plausible weather patterns, sometimes over large regions or regions with complex terrain, and thus require the capability of producing ensembles of high resolution realizations of 

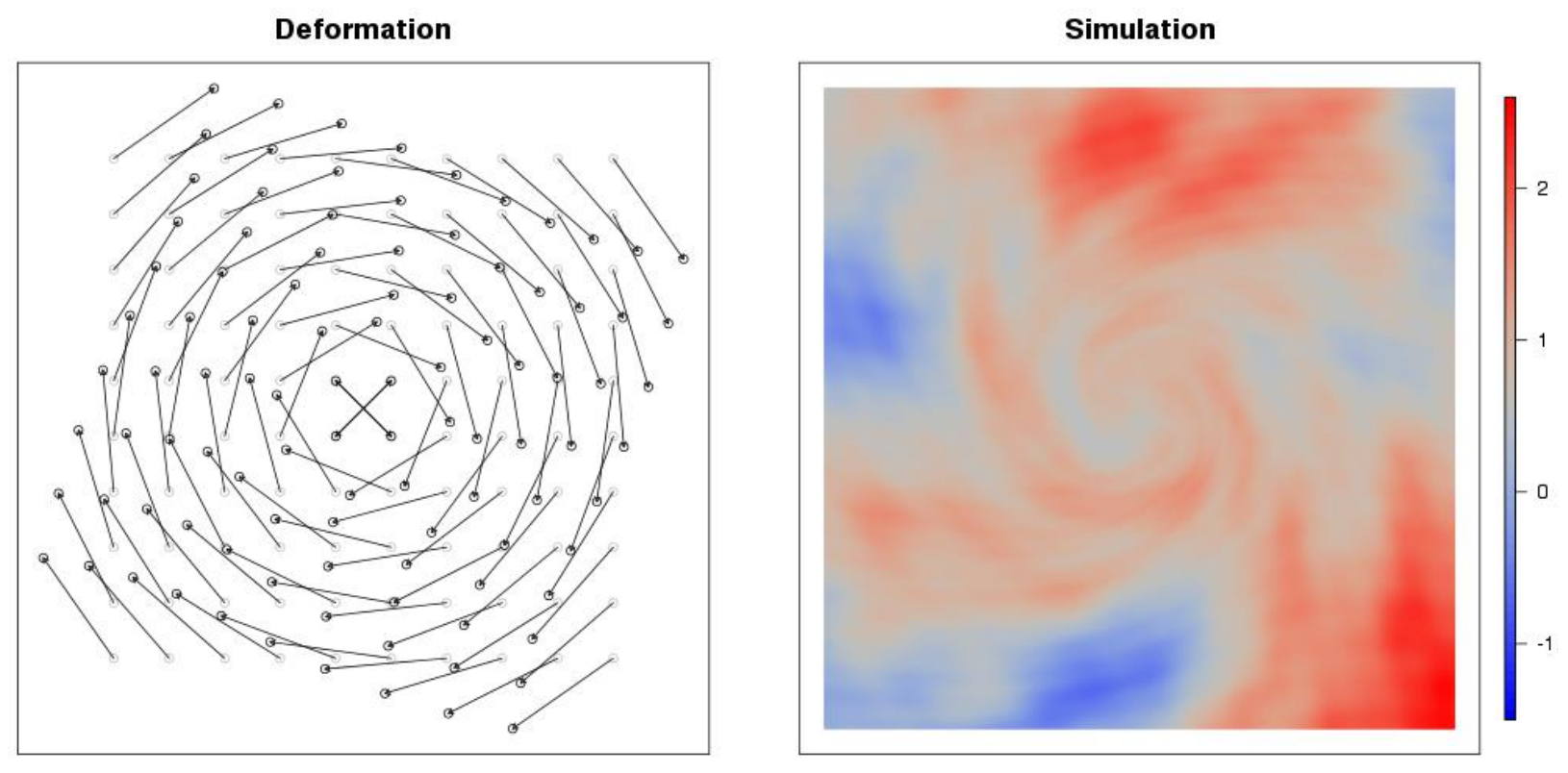

Figure 3: Vortex simulation on a $500 \times 500$ grid.

nonstationary fields. We focus here on simulating daily minimum temperature over the state of Colorado in the United States. Our observational dataset is from the United States Historical Climatology Network, with observations of minimum and maximum temperature available during 1852 through 2011 (Peterson and Vose, 1997).

As a framework for building stochastic weather generators, Kleiber et al. (2013) suggested decomposing the observations into a climate component and a weather component. We follow a similar avenue here, where we decompose minimum temperature observations $Y(\mathbf{s}, t)$ for spatial location $\mathbf{s} \in \mathbb{R}^{2}$ and time point $t$ as

$$
Y(\mathbf{s}, t)=\mu(\mathbf{s}, t)+Z(\mathbf{s}, t)+\varepsilon(\mathbf{s}, t) .
$$

Here, $\mu$ is the climatological mean function, $Z$ can be interpreted as the weather term, and $\varepsilon$ is a noise process representing measurement error. We include elevation as a covariate, 
writing $\mu(\mathbf{s}, t)=\boldsymbol{\beta}(\mathbf{s})^{\prime} \mathbf{X}(\mathbf{s}, t)$ with

$$
\mathbf{X}(\mathbf{s}, t)=\left(1, e(\mathbf{s}), \cos \left(\frac{2 \pi t}{365}\right), \sin \left(\frac{2 \pi t}{365}\right), Y(\mathbf{s}, t-1), M(\mathbf{s}, t-1), r(t)\right)^{\prime}
$$

where $e(\mathbf{s})$ is the elevation at location $\mathbf{s}, M(\mathbf{s}, t-1)$ is the previous day's maximum temperature and $r(t)$ is a linear drift term to account for possible climate change. We opt not to include higher order harmonics, following Kleiber et al. (2013), and note that the illustrations below are for individual days, where the temporal structure is less important. The corresponding model coefficients are $\boldsymbol{\beta}(\mathbf{s})=\left(\beta_{0}, \beta_{1}, \beta_{2}(\mathbf{s}), \beta_{3}(\mathbf{s}), \beta_{4}(\mathbf{s}), \beta_{5}(\mathbf{s}), \beta_{6}(\mathbf{s})\right)^{\prime}$, where the first two parameters are spatially constant. We estimate $\beta_{0}$ and $\beta_{1}$ by ordinary least squares on all available observations, followed by estimating remaining regression parameters locally at each individual observation location by ordinary least squares based on residuals from the global regression. The spatial covariance of $Z(\mathbf{s}, t)$ is estimated nonparametrically using an estimator similar to (2) applied to the residuals, except that additionally includes kernel smoothing over time, see Kleiber et al. (2013) for details.

We focus simulations on daily minimum temperature observations on June 29. The previous days temperatures are set as the climatological mean for June 28. Corresponding stationary simulations must come from a specified probability model. If the true process were stationary, the deformation function would reduce to the identity and the untransformed stationary simulation would be used. Thus, our heuristic for choosing a stationary model is to choose one that best matches the observed process. To this end, we estimate a stationary correlation function $R_{D}(\cdot)$ by minimizing $(3)$ with $\lambda=0$ and $\varphi$ set to the identity map. For the minimum temperature dataset, we choose $R_{D}$ to be a Matérn correlation function with smoothness set to unity, following North et al. (2011). 

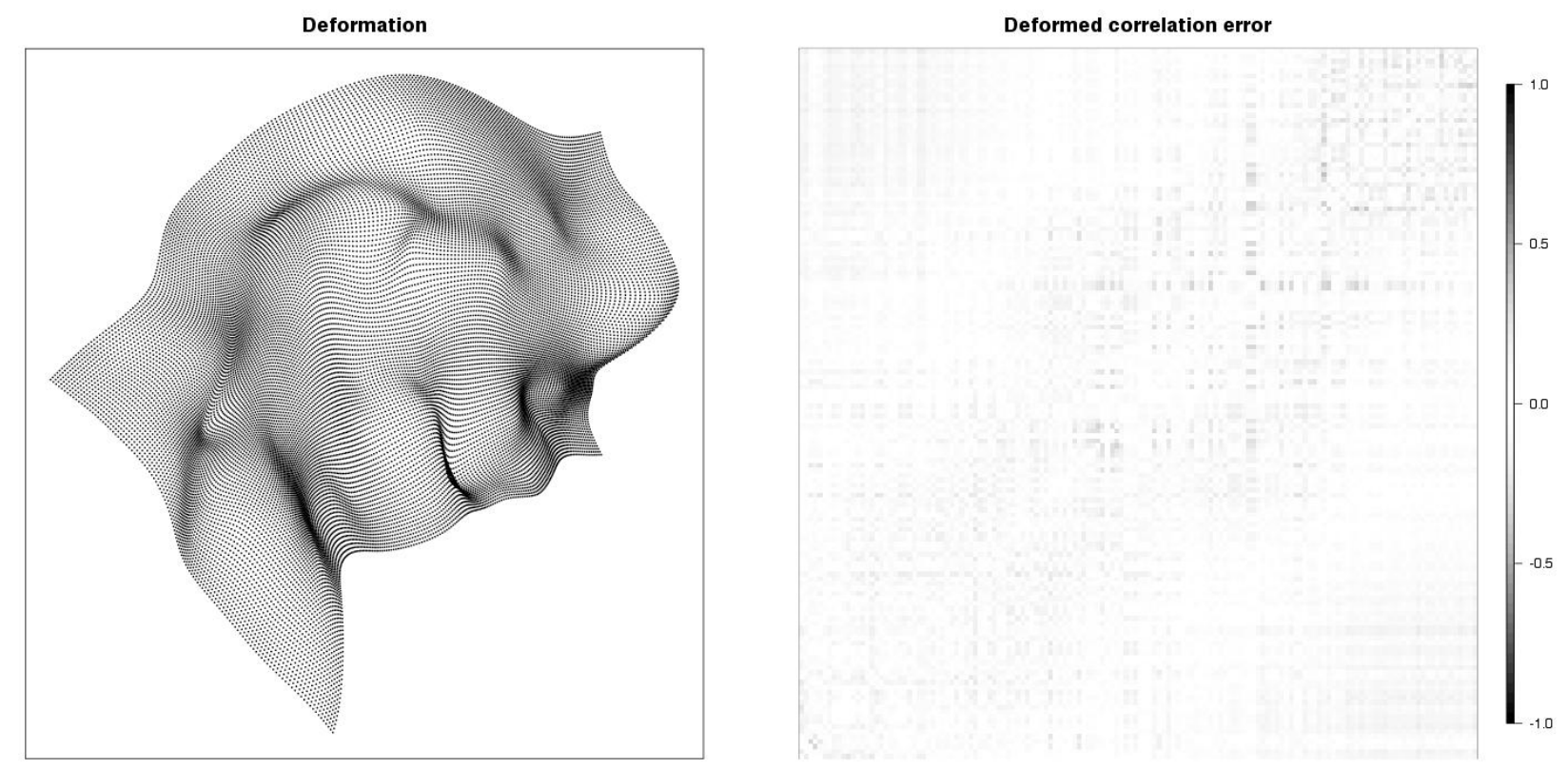

Figure 4: Estimated warping function for the minimum temperature dataset over Colorado (left panel). The right panel shows the deformed correlation error.

The deformation function is estimated as in (3) using the thin plate spline penalty formulation for the inner product and corresponding deformation function. We use an iterative procedure, first estimating the warp for three randomly chosen landmark locations, then using the predicted deformation for four locations as a starting point, estimate the deformation for those four. We iterate this procedure until 100 of the locations have been included.

The resulting deformation function is shown in Figure 4; note that the estimated deformation function suggests the presence of substantial nonstationarity in the minimum temperature residuals. Areas that appear pinched according to the deformation function will be stretched in the nonstationary simulation (thus having a greater spatial range), whereas areas that appear stretched will have greater variability in the nonstationary plane. Visually, the deformation function suggests there will be areas of stronger correlation the left and 
right side of Colorado, with more variability split down the center. This agrees well with the estimated correlation function in that there are strong correlations within the mountainous western region and plains eastern region, but less strong correlation between the two regions (see Kleiber et al. 2013 for further discussion of the local climate and spatial structure). Figure 4 also illustrates the deformed correlation error, that is, the difference in estimated nonstationary covariance (2) and the warped stationary correlation $\left(R_{D}\left(\hat{\varphi}\left(\mathbf{s}_{i}\right)-\hat{\varphi}\left(\mathbf{s}_{j}\right)\right)\right)$. The deformed stationary covariance appears to replicate with reasonable accuracy the patterns and magnitude of nonstationary covariance present in the dataset.

The ability to perform high resolution nonstationary simulations allows for quantitative assessments of features of the process that would otherwise be difficult to calculate analytically. For example, road maintenance can play a large economic role, requiring short terms decisions and long term planning (Berrocal et al., 2010). Interstate 70 runs through Colorado in a nearly east-west direction and is influenced by weather in the eastern plains, the Rocky Mountains as well as the western slopes. Figure 5 shows the path of I-70 through Colorado, as well as the local elevation along I-70. To illustrate the importance of producing nonstationary simulations, we compare 200 independent simulations using our nonstationary method and a competing stationary method using circulant embedding with a Matérn covariance function whose parameters were estimated by maximum likelihood. Figure 5 shows functional boxplots for both types of simulations (Sun and Genton, 2011). Functional boxplots are preferable here as they capture the behavior of each simulated curve and thus are not restricted to pointwise interpretations. The stationary model is able to identify the climatological trend of minimum temperature over I-70 in that the Rocky Mountains tend to 


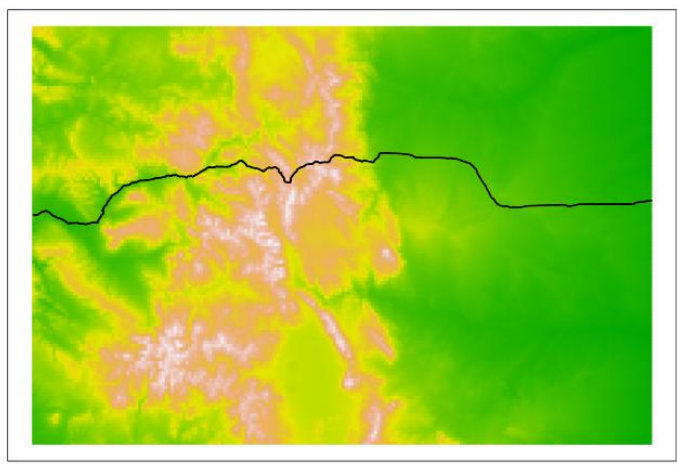

Nonstationary

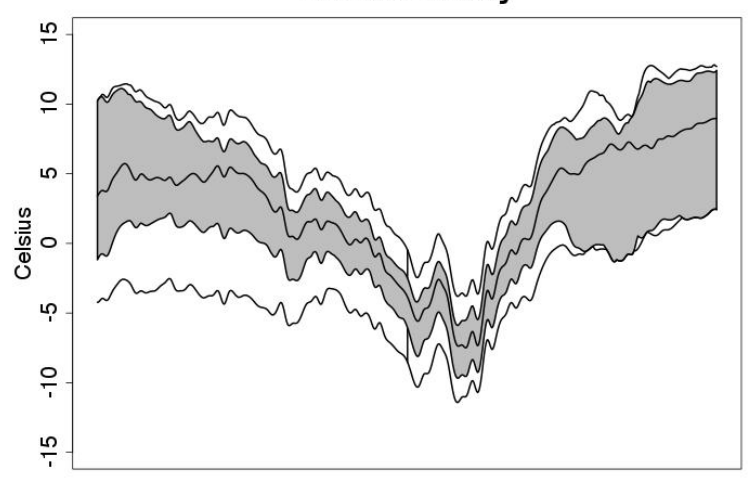

\section{Elevation}

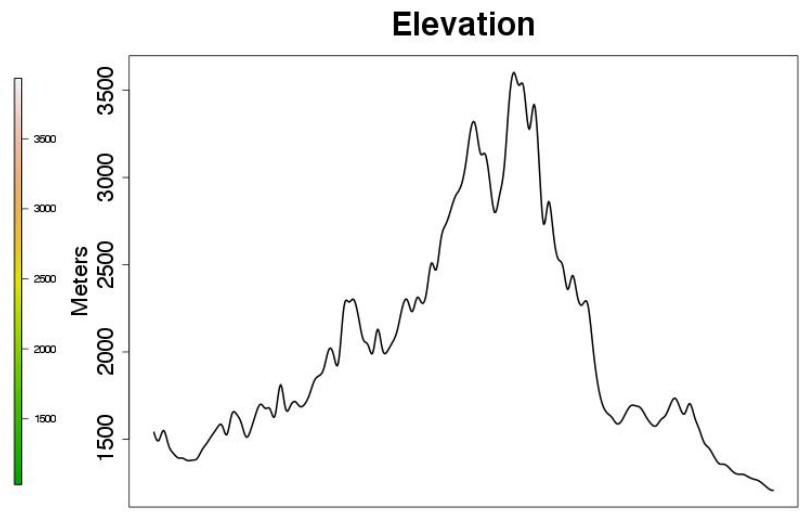

Stationary

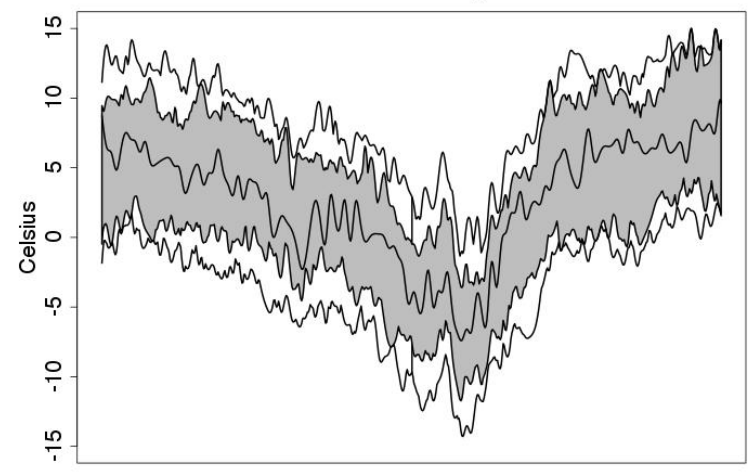

Figure 5: Simulation of minimum temperature on Interstate 70 passing through Colorado (shown black line in upper left plot over an elevation heatmap; elevations are in meters above sea level). The upper right panel shows the change in elevation along I-70. The bottom row contains functional boxplots for minimum temperature simulations along I-70 based on the proposed nonstationary method as well as a stationary Matérn model.

exhibit colder minima than the plains, however the nonstationary simulations additionally capture the change in variability of minimum temperatures, with greater variability over the western slopes and eastern plains and more consistent minima in the mountains. Thus, the nonstationary model provides a superior representation of the physical process and whose simulations are only available at high resolution using the proposed method.

For climate change impact assessment scenarios it is crucial to accurately capture the true underlying physical behavior of meteorological processes (Semenov and Barrow, 1997). Sup- 
pose interest focused on state-wide extrema of minimum temperature in Colorado, which is an important benchmark against which future climate model projections may be compared. Figure 6 shows density estimates of the $5 \%, 50 \%$ and $95 \%$ quantiles of state-wide minimum temperature based on 200 simulations from our nonstationary model and the stationary model based on the Matérn covariance (whose simulations are produced using circulant embedding); simulations are produced on a grid of 25,200 locations. The nonstationary model simulations are based on stationary plane simulation via circulant embedding at a $1000 \times 1000$ grid; simulations in the nonstationary plane are identified by their nearest neighbor to this dense stationary simulation. While both the nonstationary and stationary models capture approximately the same average behavior of these quantiles, the nonstationary model exhibits substantially different second moment behavior. In particular, at the lowest quantiles, the nonstationary simulation exhibits a standard deviation that is approximately $28 \%$ lower relative to the stationary simulation (1.27 and 1.78 degrees Celsius, respectively, for the $5 \%$ state-wide quantile), whereas both methods have similar variation at the $95 \%$ quantiles, 1.60 and 1.72 degrees Celsius, respectively. The interpretation of this is that the coldest extremal low temperatures tend to be more clustered than the mild low temperatures. Simulations based on a stationary model are unable to elucidate this climatological feature, and additionally the nonstationary simulations are infeasible to implement without the proposed methods.

Finally, we illustrate two independent simulations over the state of Colorado that highlight important and difficult nonstationary features present in the process. Figure 7 show two independent simulations of minimum temperature over the state of Colorado for June 29 at 


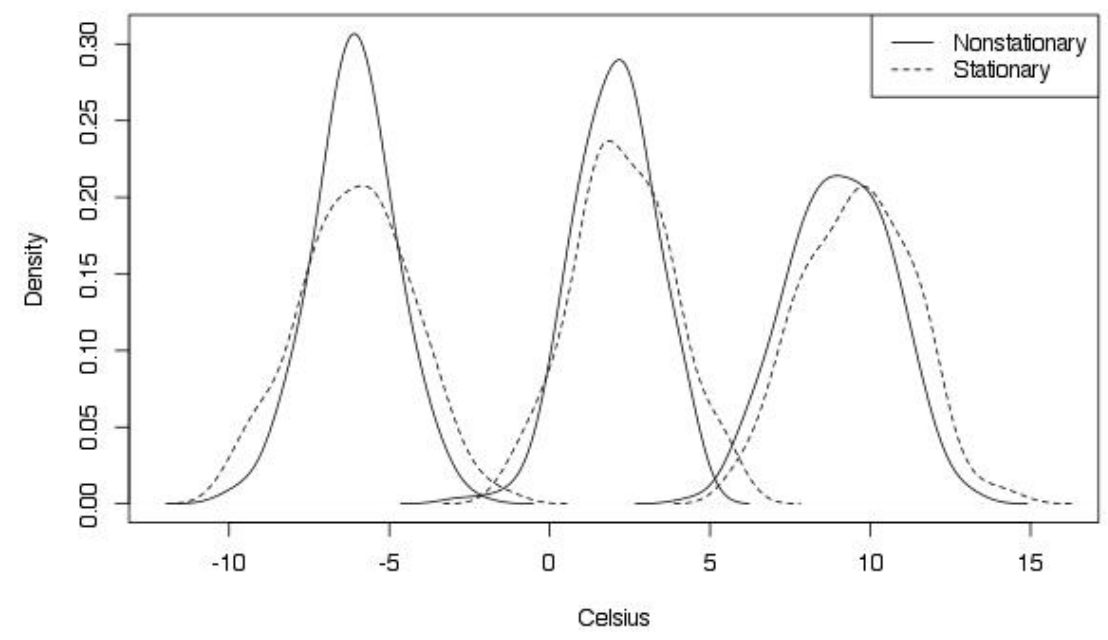

Figure 6: $5 \%, 50 \%$ and $95 \%$ quantiles of state-wide minimum temperature based on 200 independent simulations on a grid of 25,200 locations from the nonstationary model and a stationary model with Matérn covariance.

25,200 locations. Comparing the two simulations, we see that the eastern plains experience cooler weather in the second than in the first, while the mountainous Rocky region in central Colorado has approximately similar minimum temperatures. However, the western slopes experience much warmer conditions in the second simulation as compared to the first, which is a direct effect of the nonstationary correlation over the western slopes. We reiterate that such simulations would be nearly impossible to generate at such spatial resolution using a traditional matrix multiplication technique.

Regarding the total timing for estimation and simulation, estimation of the deformation function is typically the bottleneck in computations. For this example, estimating the warping function through the above iterative procedure took approximately 7 minutes in a standard implementation of R (Ihaka and Gentleman, 1996) on a MacBook Pro laptop with 
Simulation 1

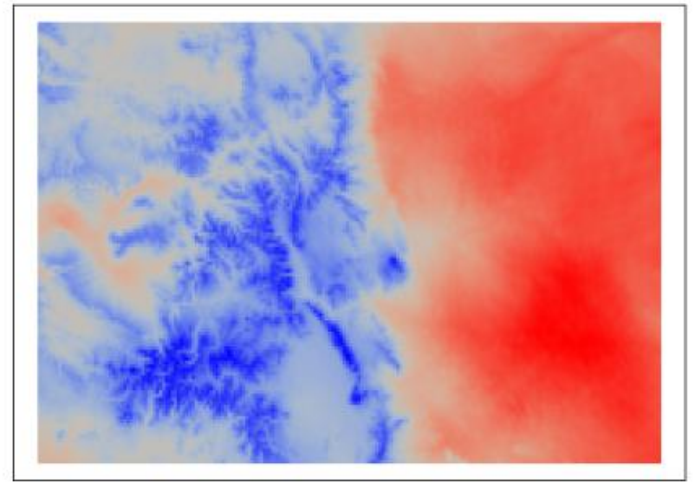

Simulation 2

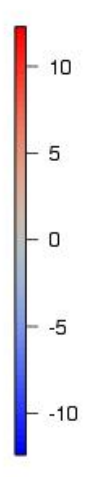

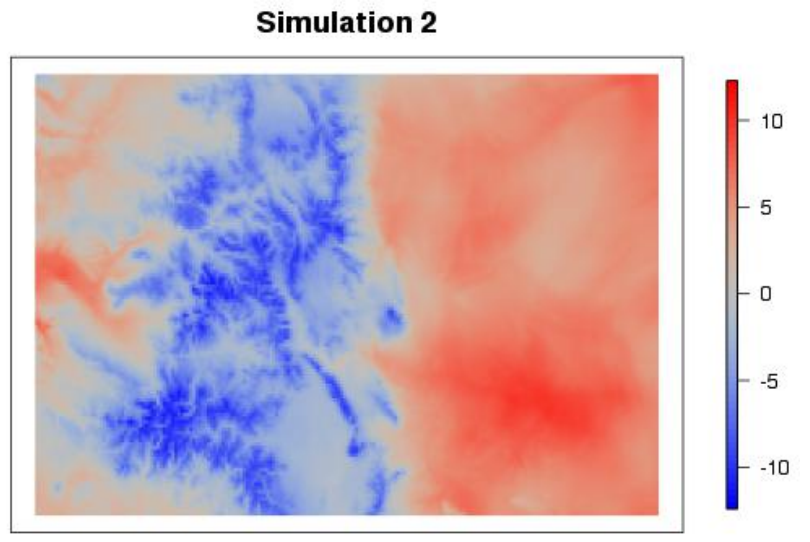

Figure 7: Two independent minimum temperature simulations for June 29 over Colorado. Simulations are realizations from a nonstationary Gaussian process and are produced on a grid of 25,200 locations; each simulation requires approximately 7.5 seconds of computation time.

a $2.9 \mathrm{GHz}$ Intel Core i7 processor and 8GB of $1600 \mathrm{MHz}$ DDR3 RAM. Once the deformation function is known, simulations are then very fast: each circulant embedding simulation on a $1000 \times 1000$ grid took approximately 7.5 seconds.

\subsection{Emulating a Regional Climate Model}

The final example we entertain is the simulation of meteorological surfaces that are consistent with regional climate model (RCM) solutions. The particular RCM we examine is the Experimental Climate Prediction Center Regional Spectral Model (ECP2) with National Center for Environmental Prediction reanalysis 2 (NCEP2) data provided as boundary conditions that was run as part of the North American Regional Climate Change Assessment Program (Mearns et al., 2009, NARCCAP). The resulting RCM run is an estimate of the historical monthly climate during the years 1981-2004.

The process we examine is average cube-root winter precipitation (DJF) over North Amer- 

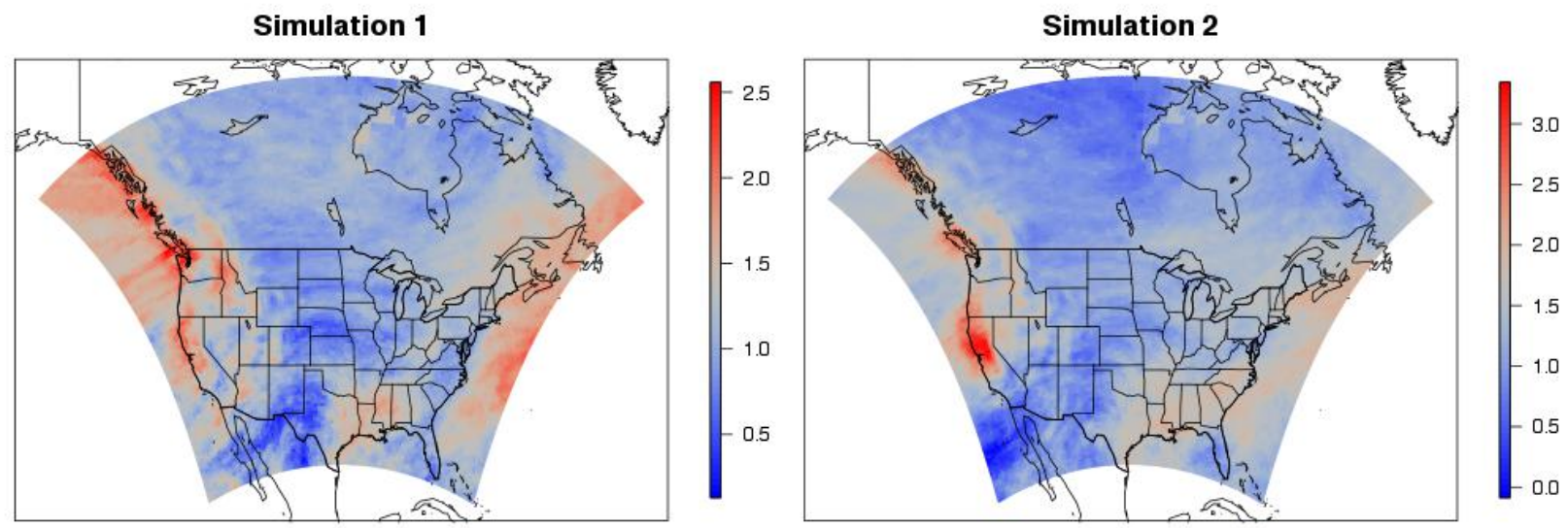

Figure 8: Two independent winter average cube-root precipitation simulations over North America. Simulations are realizations from a nonstationary Gaussian process and are produced on a grid of $120 \times 98$ locations; each simulation requires approximately 1 second of computation time.

ica. The cube-root transformation of precipitation makes the resulting field approximately Gaussian. We initially remove a spatially varying mean from the cube-root monthly precipitation estimated as the arithmetic average over all available months of winter data. The spatial covariance matrix is then estimated by averaging all empirical covariance matrices over all available months. The stationary process from which simulations will be generated is set to be a mean zero Gaussian process with an exponential covariance; we use the exponential as precipitation tends to exhibit rough characteristics over space and is highly influenced by local geographical characteristics such as mountains and coastal regions. We use a randomly chosen set of 108 grid points (about $1 \%$ of the total number of grid points) as landmark locations, but perform estimation of the deformation function in an iterative fashion: the initial estimation is done over a set of 3 locations, and then individual locations are included in a stepwise fashion until all 108 have been optimized. The initial estimations are very fast due to the small number of entertained landmarks, while the later optimizations 
are more time consuming, typically taking on the order of a few minutes to optimize.

This particular RCM is generated at a grid of $120 \times 98$ cells; simulating from a general nonstationary Gaussian process, say one with a nonstationary version of the Matérn covariance, would be difficult given the dimensionality of the climate model. Figure 8 shows two nonstationary simulations that were generated by deforming a stationary simulation to the nonstationary plane. In particular, the stationary simulations are produced via circulant embedding at a grid of $500 \times 500$ locations, and the nonstationary values are found via the nearest neighbors of the undeformed NARCCAP grid; each resulting simulation takes approximately 1 second to compute. In Figure 8, notice the principal types of nonstationarity that are apparent in these simulations - greater correlation of precipitation over the Atlantic ocean and the so-called pineapple express phenomenon in the northern Pacific ocean that gives rise to the extreme precipitation events seen in the Pacific Northwest (Weller and Cooley, 2012). These physical phenomena would not be present in a stationary simulation.

Climate change assessments require both estimates of future and past climate states. In particular, historical climate states are uncertain due to incomplete and noisy observations, and the ability to emulate climate models constrained by observational data with accurate descriptions of the spatial behavior is crucial for estimating the uncertainty in historical climate quantities. For instance, interest often focuses on how the average behavior of a process changes over time, but an equally important (or perhaps moreso) quantity is precipitation's behavior in the tails. We examine the domain-wide North American median and $95 \%$ quantile of precipitation over 200 independent nonstationary simulations; the corresponding densities are shown in Figure 9. Evidently, there is greater variability in the high 


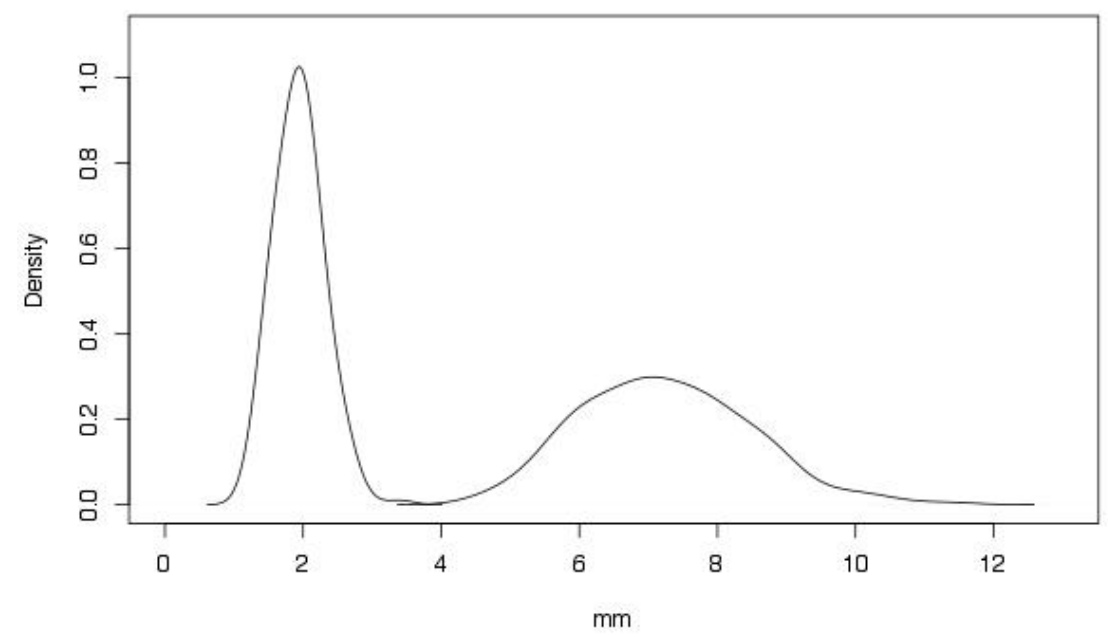

Figure 9: Density estimates of the North American 50\% and 95\% quantiles of precipitation (in $\mathrm{mm}$ ) based on 200 independent simulations on a grid of 11,760 based on a nonstationary emulator of a NARCCAP component climate model.

precipitation events than in the spatial median, and thus any climate change assessment comparison to these higher quantiles should account for this discrepancy in the variation in estimated historical climate. For example, our estimate of the spatial maximum of North American precipitation is $22.7 \mathrm{~mm}$ with a $95 \%$ confidence interval of $(13.5,39.5)$ based on our simulations. Such information is difficult to derive from ensembles of climate model runs given their well known underdispersion, and thus requires a flexible framework for statistical emulation and simulation.

\section{Discussion}

This manuscript focuses on an approach to generating high resolution nonstationary random field simulations. By exploiting a deformation function linking the nonstationary process to a 
deformed stationary process, simulations can proceed by utilizing well-known and established stationary simulation methods followed by a transformation to the nonstationary plane. Local variances are extracted, which allow for spatially-varying scale behavior. Estimation is separated into two steps. The first involves estimation of the nonstationary covariance function, for which we use a nonparametric estimator. The second estimation step is to find the deformation function, which follows from a straightforward regularized minimum distance estimator from the estimated nonstationary correlation.

The first scientific motivation for our proposal is in the field of stochastic weather simulation, where a fundamental goal is to simulate plausible weather scenarios at high resolutions that can be used for climate model downscaling or observational network infilling. The example of daily minimum temperature simulation over the state of Colorado provides challenging obstacles for any simulation method, and our example suggests we are able to maintain physically reasonable behavior in the simulated nonstationary fields. We additionally showed that stationary simulations do not provide adequate representations of the process, thus underscoring the need for a flexible nonstationary simulation framework. We secondly considered generating stochastic realizations of spatial fields that are consistent with regional climate model output. Our example simulations show physical behavior consistent with the NARCCAP RCM runs, exhibiting pineapple express type events, as well as long range correlations over the Atlantic ocean for precipitation. Spatial quantiles are readily estimated with uncertainty from the simulations, providing a feasible approach for uncertainty quantification in climate projections.

Extensions to our deformed simulation approach are called for, including the difficult 
possibility of simultaneous space-time simulation. High resolution multivariate simulations are also desirable, as well as exploring other classes of deformation functions that may yield different types of flexibility than the thin plate spline approach explored herein.

\section{Acknowledgements}

The author wishes to thank Michael Scheuerer for helpful discussions during the development of this work. This research was supported by National Science Foundation grants BCS1461576, DMS-1406536 and DMS-1417724.

\section{References}

Anderes, E. and Chatterjee, S. (2009), "Consistent estimates of deformed isotropic Gaussian random fields on the plane," Annals of Statistics, 37, 2324-2350.

Anderes, E. B. and Stein, M. L. (2008), "Estimating deformations of isotropic Gaussian random fields on the plane," Annals of Statistics, 36, 719-741.

Barndorff-Nielsen, O. E. and Shepard, N. (2006), "Impact of jumps on returns and realised variances: econometric analysis of time-deformed Lévy processes," Journal of Econometrics, 131, 217-252.

Berrocal, V. J., Raftery, A. E., Gneiting, T., and Steed, R. C. (2010), "Probabilistic weather forecasting for winter road maintenance," Journal of the American Statistical Association, $105,522-537$. 
Bookstein, F. L. (1989), "Principal warps: thin-plate splines and the decomposition of deformations," IEEE Transactions on Pattern Analysis and Machine Intelligence, 11, 567-585.

Brown, P. J., Le, N. S., and Zidek, J. V. (1994), "Multivariate spatial interpolation and exposure to air pollutants," Canadian Journal of Statistics, 22, 489-509.

Chilès, J. P. and Delfiner, P. (1999), Geostatistics: Modeling Spatial Uncertainty, New York: Wiley.

Damian, D., Sampson, P. D., and Guttorp, P. (2001), "Bayesian estimation of semiparametric non-stationary spatial covariance structures," Environmetrics, 12, 161-178.

Deodatis, G. (1996), "Nonstationary stochastic vector processes: seismic ground motion applications," Probabilistic Engineering Mechanics, 11, 149-168.

Deodatis, G. and Micaletti, R. C. (2001), "Simulation of highly skewed non-Gaussian stochastic processes," Journal of Engineering Mechanics, 127, 1284-1295.

Dietrich, C. R. (1993), "Computationally efficient Cholesky factorization of a covariance matrix with block Toeplitz structure," Journal of Statistical and Computation and Simulation, 45, 203-218.

Dietrich, C. R. (1995), "A simple and efficient space domain implementation of the turning bands method," Water Resources Research, 31, 147-156.

Dietrich, C. R. and Newsam, G. N. (1993), "A fast and exact method for multidimensional Gaussian stochastic simulations," Water Resources Research, 29, 2861-2869. 
Dietrich, C. R. and Newsam, G. N. (1997), "Fast and exact simulation of stationary Gaussian processes through circulant embedding of the covariance matrix," SIAM Journal on Scientific Computing, 18, 1088-1107.

Ehm, W., Gneiting, T., and Richards, D. (2004), "Convolution roots of radial positive definite functions with compact support," Transactions of the American Mathematical Society, 356, 4655-4685.

Ghanem, R. and Spanos, P. (1990), "Polynomial chaos in stochastic finite elements," Journal of Applied Mechanics, 57, 197-202.

Gilleland, E., Chen, L., DePersio, M., Do, G., Eilertson, K., Jin, Y., Kang, E. L., Lindgren, F., Lindström, J., Smith, R. L., and Xia, C. (2011), "Spatial forecast verification: Image warping," Tech. Rep. TN-482+STR, National Center for Atmospheric Research.

Gneiting, T. (1996), "Comment on 'A simple and efficient space domain implementation of the turning bands method' by C. R. Dietrich," Water Resources Research, 32, 3391-3396.

Gneiting, T. (1998), "Closed form solutions of the two-dimensional turning bands equation," Mathematical Geology, 30, 379-390.

Gneiting, T. (1999), "The correlation bias for two-dimensional simulations by turning bands," Mathematical Geology, 31, 195-211.

Gneiting, T., Ševčíková, H., Percival, D. B., Schlather, M., and Jiang, Y. (2006), "Fast and exact simulations of large Gaussian lattice systems in $\mathbb{R}^{2}$ : exploring the limits," Journal of Computational and Graphical Statistics, 15, 483-501. 
Green, P. J. and Silverman, B. W. (1994), Nonparametric Regression and Generalized Linear Models: A Roughness Penalty Approach, Boca Raton: Chapman \& Hall/CRC.

Guillot, G., Senoussi, R., and Monestiez, P. (2001), "A positive definite estimator of the non stationary covariance of random fields," in GeoENV 2000: Third European Conference on Geostatistics for Environmental Applications, eds. P. Monestiez, D. Allard, and R. Froidevaux, Kluwer Academic Publishers, Dordrecht, the Netherlands.

Guttorp, P. and Sampson, P. D. (1994), "Methods for estimating heterogeneous spatial covariance functions with environmental applications," in Handbook of Statistics XII: Environmental Statistics, eds. G. P. Patil and C. R. Rao, pp. 663-690, Elsevier/North-Holland, Amsterdam.

Guttorp, P., Sampson, P. D., and Newman, K. (1992), "Nonparametric estimation of spatial covariance with application to monitoring network evaluation," in Statistics in the Environmental and Earth Sciences, eds. P. Guttorp and A. Walden, pp. 39-57, Edward Arnold Press, London.

Guttorp, P., Meiring, W., and Sampson, P. D. (1994), "A space-time analysis of ground-level ozone data," Environmetrics, 5, 241-254.

Huang, S. P., Quek, S. T., and Phoon, K. K. (2001), "Convergence study of the truncated Karhunen-Loeve expansion for simulation of stochastic processes," International Journal for Numerical Methods in Engineering, 52, 1029-1043. 
Ihaka, R. and Gentleman, R. (1996), "R: A language for data analysis and graphics," Journal of Computational and Graphical Statistics, 5, 299-314.

Iovleff, S. and Perrin, O. (2004), "Estimating a nonstationary spatial structure using simulated annealing," Journal of Computational and Graphical Statistics, 13, 90-105.

Journel, A. G. (1974), "Geostatistics for conditional simulation of ore bodies," Economic Geology, 69, 673-687.

Jun, M., Szunyogh, I., Genton, M. G., Zhang, F., and Bishop, C. H. (2011), "A statistical investigation of the sensitivity of ensemble-based Kalman filters to covariance filtering," Monthly Weather Review, 139, 3036-3051.

Kleiber, W. and Nychka, D. (2012), "Nonstationary modeling for multivariate spatial processes," Journal of Multivariate Analysis, 112, 76-91.

Kleiber, W., Katz, R. W., and Rajagopalan, B. (2013), "Daily minimum and maximum temperature simulation over complex terrain," Annals of Applied Statistics, 7, 588-612.

Kroese, D. P. and Botev, Z. I. (2013), "Spatial process generation," in Lectures on Stochastic Geometry, Spatial Statistics and Random Fields, Volume II: Analysis, Modeling and Simulation of Complex Structures, ed. V. Schmidt, Springer-Verlag, Berlin.

Lantuéjoul, C. and Desassis, N. (2012), "Simulation of a Gaussian random vector: A propagative version of the Gibbs sampler," in The 9th International Geostatistics Congress.

Li, Y. and Kareem, A. (1991), "Simulation of multivariate nonstationary random processes by FFT," Journal of Engineering Mechanics, 117, 1037-1058. 
Liang, J., Chaudhuri, S. R., and Shinozuka, M. (2007), "Simulation of nonstationary stochastic processes by spectral representation," Journal of Engineering Mechanics, 2007, 616627.

Lindgren, F., Rue, H., and Lindström, J. (2011), "An explicit link between Gaussian fields and Gaussian Markov random fields: the stochastic partial differential equation approach," Journal of the Royal Statistical Society, Series B, 73, 423-498.

Mantoglou, A. and Wilson, J. L. (1982), "The turning bands method for simulation of random fields using line generation by a spectral method," Water Resources Research, 18, $1379-1394$.

Matheron, G. (1973), "The intrinsic random functions and their applications," Advances in Applied Probability, 5, 439-468.

Mearns, L. O., Gutowski, W. J., Jones, R., Leung, L., McGinnis, A. M., Nunes, B., and Qian, Y. (2009), "A regional climate change assessment program for North America," Eos Transactions, American Geophysical Union, 90, 311-312.

Meiring, W. (1995), "Estimation of heterogeneous space-time covariance," Ph.D. thesis, University of Washington, Seattle.

Meiring, W., Monestiez, P., Sampson, P. D., and Guttorp, P. (1997), "Developments in the modelling of nonstationary spatial covariance structure from space-time monitoring data," in Geostatistics Wollongong '96, Volume 1, eds. E. Baaffi and N. Schofield, pp. 162-173, Kluwer Academic Publishers, Dordrecht. 
Meiring, W., Guttorp, P., and Sampson, P. D. (1998), "Space-time estimation of grid-cell hourly ozone levels for assessment of a deterministic model," Environmental and Ecological Statistics, 5, 197-222.

Monestiez, P., Sampson, P. D., and Guttorp, P. (1993), "Modelling of heterogeneous spatial correlation structure by spatial deformation," Cahiers de Geostatistique, Fascicule 3, Compte Rendu des Journees de Geostatistique.

North, G. R., Wang, J., and Genton, M. G. (2011), "Correlation models for temperature fields," Journal of Climate, 24, 5850-5862.

Nychka, D., Bandyopadhyay, S., Hammerling, D., Lindgren, F., and Sain, S. (2014), "A multi-resolution Gaussian process model for the analysis of large spatial data sets," Journal of Computational and Graphical Statistics, in press.

Oehlert, G. W. (1993), "Regional trends in sulfate wet deposition," Journal of the American Statistical Association, 88, 390-399.

Paciorek, C. J. and Schervish, M. J. (2006), "Spatial modelling using a new class of nonstationary covariance functions," Environmetrics, 17, 483-506.

Perrin, O. and Meiring, W. (1999), "Identifiability for non-stationary spatial structure," Journal of Applied Probability, 36, 1244-1250.

Perrin, O. and Monestiez, P. (1998), "Modelling of non-stationary spatial structure using parametric radial basis deformations," in geoENV98, eds. A. Soares, J. Gómez-Hernandez, and R. Froidevaux, pp. 175-186, Amsterdam: Kluwer Academic Publishers. 
Perrin, O. and Senoussi, R. (1999), "Reducing non-stationary stochastic processes to stationarity by a time deformation," Statistics and Probability Letters, 43, 393-397.

Perrin, O. and Senoussi, R. (2000), "Reducing non-stationary random fields to stationarity and isotropy using a space deformation," Statistics and Probability Letters, 48, 23-32.

Peterson, T. C. and Vose, R. S. (1997), "An overview of the Global Historical Climatology Network temperature database," Bulletin of the American Meteorological Society, 78, 2837-2849.

Puig, B., Poirion, F., and Soize, C. (2002), "Non-Gaussian simulation using Hermite polynomial expansion: convergences and algorithms," Probabilistic Engineering Mechanics, 17, 253-264.

Reich, B. J. and Fuentes, M. (2007), “A multivariate semiparametric Bayesian spatial modeling framework for hurricane surface wind fields," Annals of Applied Statistics, 1, 249-264.

Sakamoto, S. and Ghanem, R. (2002a), "Polynomial chaos decomposition for the simulation of non-Gaussian nonstationary stochastic processes," Journal of Engineering Mechanics, $128,190-201$.

Sakamoto, S. and Ghanem, R. (2002b), "Simulation of multi-dimensional non-gaussian nonstationary random fields," Probabilistic Engineering Mechanics, 17, 167-176.

Sampson, P. D. (2010), "Constructions for nonstationary spatial processes," in Handbook of Spatial Statistics, eds. A. E. Gelfand, P. Diggle, P. Guttorp, and M. Fuentes, pp. 119-130, Boca Raton: Chapman \& Hall / CRC. 
Sampson, P. D. and Guttorp, P. (1992), "Nonparametric estimation of nonstationary spatial covariance structure," Journal of the American Statistical Association, 87, 108-119.

Sarma, P., Durlofsky, L. J., and Aziz, K. (2008), "Kernel principal component analysis for efficient, differentiable parameterization of multipoint geostatistics," Mathematical Geosciences, 40, 3-32.

Schlather, M. (2012), "Construction of covariance functions and unconditional simulations of random fields," in Advances and Challenges in Space-time Modelling of Natural Events, eds. E. Porcu, J. Montero, and M. Schlather, pp. 25-54, Springer-Verlag: Berlin, Heidelberg.

Schlather, M., Malinowski, A., Oesting, M., Boecker, D., Strokorb, K., Engelke, S., Martini, J., Ballani, F., Menck, P. J., Gross, S., Ober, U., Burmeister, K., Manitz, J., Ribeiro, P., Singleton, R., Pfaff, B., and R Core Team (2014), RandomFields: Simulation and Analysis of Random Fields, R package version 3.0.44.

Semenov, M. A. and Barrow, E. M. (1997), "Use of a stochastic weather generator in the development of climate change scenarios," Climatic Change, 35, 397-414.

Serinaldi, F. and Kilsby, C. G. (2014), "Simulating daily rainfall fields over large areas for collective risk estimation," Journal of Hydrology, 512, 285-302.

Shinozuka, M. and Jan, C. M. (1972), "Digital simulation of random processes and its applications," Journal of Sound and Vibration, 25, 111-128. 
Soize, C. and Ghanem, R. (2004), "Physical systems with random uncertainties: chaos representations with arbitrary probability measure," SIAM Journal on Scientific Computing, $26,395-410$.

Spanos, P. D., Beer, M., and Red-Horse, J. (2007), "Karhunen-Loéve expansion of stochastic processes with a modified exponential covariance kernel," Journal of Engineering Mechanics, $133,773-779$.

Stein, M. L. (2001), "Local stationarity and simulation of self-affine intrinsic random functions," IEEE Transactions on Information Theory, 47, 1385-1390.

Stein, M. L. (2002), "Fast and exact simulation of fractional Brownian surfaces," Journal of Computational and Graphical Statistics, 11, 587-599.

Stein, M. L. (2005), "Nonstationary spatial covariance functions," University of Chicago, CISES Technical Report 21.

Sun, Y. and Genton, M. G. (2011), "Functional boxplots," Journal of Computational and Graphical Statistics, 20, 316-334.

Wahba, G. (1990), Spline Models for Observational Data, Society for Industrial and Applied Mathematics, Philadelphia, PA.

Weller, G. B. and Cooley, D. S. (2012), "An investigation of the pineapple express phenomenon via bivariate extreme value theory," Environmetrics, 23, 420-439.

Wilks, D. S. and Wilby, R. L. (1999), "The weather generation game: a review of stochastic weather models," Progress in Physical Geography, 23, 329-357. 
Wood, A. T. A. and Chan, G. (1994), "Simulation of stationary Gaussian processes in [0, 1] ," Journal of Computational and Graphical Statistics, 3, 409-432. 